\title{
Isotropically Polarized Speckle Patterns
}

\author{
Mikolaj K. Schmidt, ${ }^{1,2, *}$ Javier Aizpurua, ${ }^{1,2}$ Xavier Zambrana-Puyalto, ${ }^{3,4}$ Xavier Vidal, ${ }^{3}$ \\ Gabriel Molina-Terriza, ${ }^{3,4}$ and Juan José Sáenz ${ }^{2,5, \uparrow}$ \\ ${ }^{1}$ Materials Physics Center CSIC-UPV/EHU, Paseo Manuel de Lardizabal 5, 20018 Donostia-San Sebastián, Spain \\ ${ }^{2}$ Donostia International Physics Center DIPC, Paseo Manuel de Lardizabal 4, 20018 Donostia-San Sebastián, Spain \\ ${ }^{3}$ Department of Physics and Astronomy, Macquarie University, North Ryde, New South Wales 2109, Australia \\ ${ }^{4}$ ARC Center for Engineered Quantum Systems, Macquarie University, North Ryde, New South Wales 2109, Australia \\ ${ }^{5}$ Departamento de Física de la Materia Condensada, Instituto Nicolás Cabrera and Condensed Matter Physics Center (IFIMAC), \\ Universidad Autónoma de Madrid, 28049 Madrid, Spain \\ (Received 29 September 2014; revised manuscript received 22 December 2014; published 20 March 2015)
}

\begin{abstract}
The polarization of the light scattered by an optically dense and random solution of dielectric nanoparticles shows peculiar properties when the scatterers exhibit strong electric and magnetic polarizabilities. While the distribution of the scattering intensity in these systems shows the typical irregular speckle patterns, the helicity of the incident light can be fully conserved when the electric and magnetic polarizabilities of the scatterers are equal. We show that the multiple scattering of helical beams by a random dispersion of "dual" dipolar nanospheres leads to a speckle pattern exhibiting a perfect isotropic constant polarization, a situation that could be useful in coherent control of light as well as in lasing in random media.
\end{abstract}

DOI: 10.1103/PhysRevLett.114.113902

PACS numbers: 42.25.Dd, 42.25.Fx, 42.25.Ja, 78.67.Bf

The scattering of light by random media produces complex, irregular intensity distributions known as speckle patterns $[1,2]$. Although the study of the statistical properties of speckle patterns has been a topic of high interest during recent decades, the statistics of the polarization of electromagnetic vector waves is still not well understood. Still, the depolarization of light in a random medium is the basis of an increasingly broad range of applications from remote sensing [3], enhanced backscattering phenomena [4-7], or dynamic spectroscopy [8-10], to biomedical imaging and diagnostics [11-13]. Even for static samples, the polarization of the scattered field is far from being isotropic [3] and the polarization of the speckle pattern may exhibit rapid changes from one speckle grain to another [14] with a nontrivial statistical distribution of polarization singularities $[15,16]$.

It is generally assumed that multiple scattering of light from inhomogeneities in optically dense media randomizes the state of polarization of light. A wave propagating in such a medium becomes rapidly depolarized in a characteristic length scale that depends on the properties of both the scattering medium and the illuminating light [17-19]. Here we discuss a peculiar combination of random samples and laser beams that lead to unusual "anomalously" polarized speckle patterns exhibiting isotropic constant polarization.

Dielectric nanospheres of moderate permittivity like silicon [20-22] present strong magnetic and electric dipolar resonances in the visible, as well as in telecom and nearinfrared, frequencies, without spectral overlap between quadrupolar and higher-order modes. The interference between the electric and magnetic dipolar fields can lead to strongly asymmetric angular distributions of scattered intensity, including zero backscattering at specific wavelengths [23-27] — the so-called first Kerker condition [28]. In the dipolar approximation, such particles can be understood as "dual" scatterers, i.e., particles which are invariant under electromagnetic duality transformations. It should be noted that these scatterers are not dual in terms of the ratio of their permittivity and permeability being equal to that of the surrounding medium $[29,30]$. Instead, the duality arises when the electric and magnetic polarizabilities are identical [31]. Then, the absence of backscattered light can be understood as a direct consequence of the simultaneous conservation of angular momentum and "helicity" in the scattering from cylindrically symmetric dual particles [32].

In this Letter, we study the light scattering on dielectric dual nanospheres illuminated by Bessel beams with a welldefined component of angular and linear momenta along the axis, and helicity [29,30]. Specifically, we analyze the far-field angular intensity distribution and polarization of the scattered light on a Si dipolar nanosphere as a function of its displacement from the axis. At the dual, first Kerker, condition, far-field light polarization is independent of the scattering angle, illustrating the conservation of the helicity in scattering by a single dual nanoparticle. Furthermore, we discuss the generalization of these results to systems comprising dimers [33] and random ensembles of dual nanoparticles. Contrary to intuition, we show that the multiple scattering of helical beams by a random dispersion of dual dipolar nanospheres leads to a speckle pattern exhibiting a perfect isotropic constant polarization.

Helical beams are a special class of solutions of the freespace Maxwell equations with the well-defined wave number $k$ and helicity $\Lambda$. The latter condition can be 
written down explicitly through the representation of the helicity operator $\boldsymbol{\Lambda}$,

$$
\Lambda \mathbf{E}=\frac{1}{k} \nabla \times \mathbf{E}=\Lambda \mathbf{E},
$$

with the allowed eigenvalues of $\Lambda= \pm 1$. One elementary solution to this set of equations takes the form of a circularly polarized plane wave, for which the helicity can be identified with its handedness, giving $\Lambda=1(-1)$ for the left- (right-) hand circularly polarized light. Furthermore, if the arbitrary helical beam is decomposed into a set of plane waves, all of them will exhibit circular polarization with identical handedness.

In this work, when considering scattering into the far field, we will limit ourselves to investigating the handedness of the scattered field. This simplification stems from a simple observation: any possible detector of scattered light will be measuring the properties of a single plane wave, or a very narrow distribution of plane waves, scattered toward the detector's aperture.

However, before we consider scattering of helical beams, let us point out a very useful and crucial characteristic of such beams. Using the Faraday equation in frequency domain, we can relate the magnetic field of the beam with the curl, or-through the definition of the helicity operator-the helicity of the electric field,

$$
\mathbf{H}=-\frac{i}{\mu_{0} c} \mathbf{\Lambda} \mathbf{E}=-\frac{i}{\mu_{0} c} \Lambda \mathbf{E},
$$

where $\mu_{0}$ is the vacuum permeability and $c$ is the speed of light in vacuum.

For illustrative purposes, throughout the Letter we will be using a special form of the helical beams with an axial symmetry around the propagation direction $\hat{z}$, as defined in Ref. [30]. We should emphasize that, although throughout this work we use a specific form of the incident helical light to illustrate the scattering processes, all of the results discussed in the Letter are general and hold true for any given helical incident beam, for instance for Bessel beams of order $n \neq 1$.

The intensity of the electric field of our helical beam, plotted in the schematic of Fig. 1(a), is invariant with respect to translation along $\hat{z}$. The spacing of the fringes in the intensity cross section and its actual shape is determined by the helicity of the beam (here fixed as $\Lambda=-1$ ), its order $(n=1)$, and by the aperture angle $\left(\theta_{k}=\pi / 4\right)$ [30]. As we mentioned above, in the scattering process, the helicity of light can be associated with the handedness of the scattered plane wave. Therefore, to quantify the degree of helicity of the light scattered in the direction given by two angles $\varphi$ and $\theta$, we define a degree of helical polarization $\eta \in[-1,1]$,

$$
\eta(\varphi, \theta)=\frac{I_{\mathrm{RC}}(\varphi, \theta)-I_{\mathrm{LC}}(\varphi, \theta)}{I_{\mathrm{RC}}(\varphi, \theta)+I_{\mathrm{LC}}(\varphi, \theta)},
$$

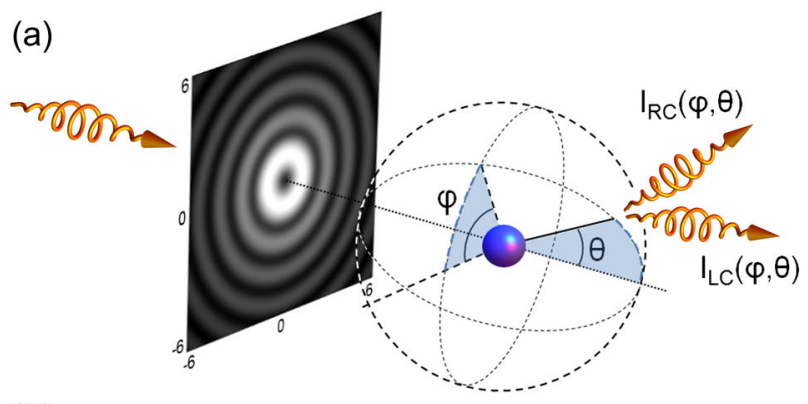

(b)
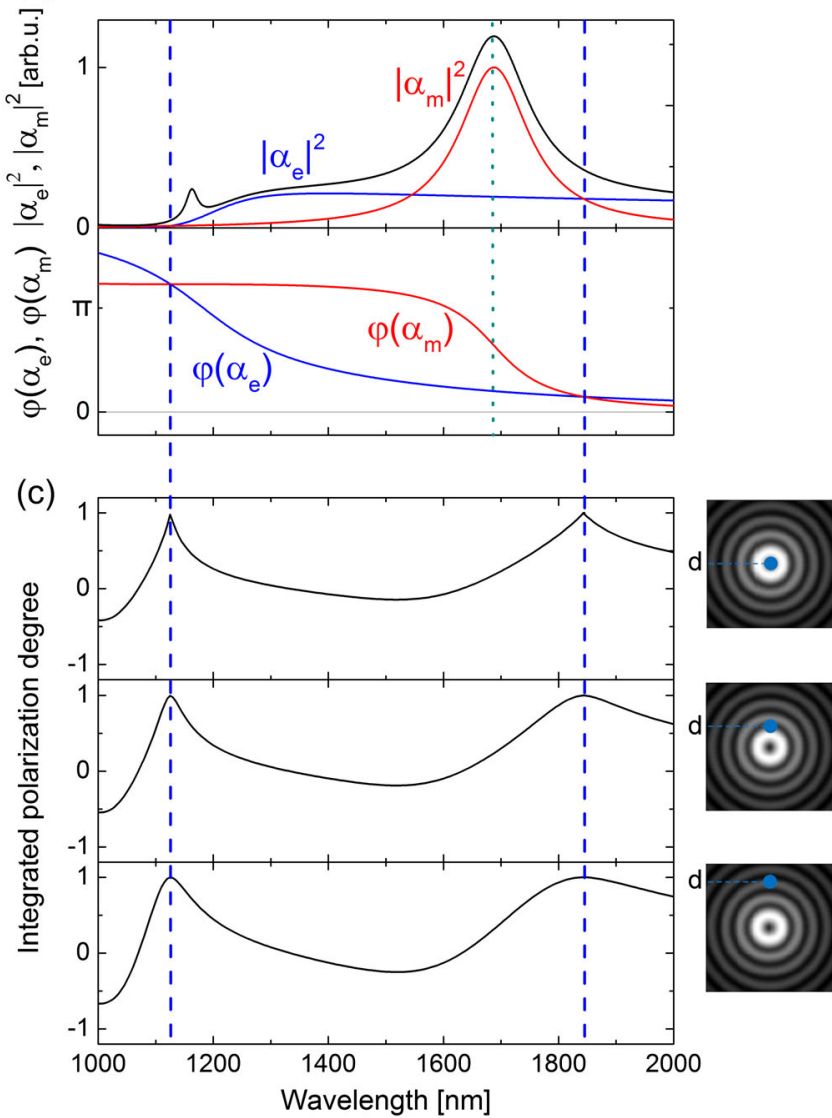

FIG. 1 (color online). (a) Schematic of the scattering process. (b) Square moduli (top panel) and phases (bottom panel) of the electric $\left(\alpha_{e}\right.$, blue line $)$ and magnetic $\left(\alpha_{m}\right.$, red line) polarizabilities of a 230-nm-radius silicon sphere in the near-IR. Scattering cross section $\left(\sigma_{\text {scatt }} k^{-4}\right.$ shown with black line) is dominated by the contributions from these dipolar terms. Dashed vertical lines indicate wavelengths at which the polarizabilities are equal both in magnitude and phase. (c) Integrated circular polarization factors $\eta_{\text {tot }}$ for the sphere positioned on the axis of the beam (top panel), and displaced by $2 \mu \mathrm{m}$ (middle panel) and $4 \mu \mathrm{m}$ (bottom panel) from the axis, as shown in the insets.

where $I_{\mathrm{RC}}$ and $I_{\mathrm{LC}}$ are the polarization-resolved differential scattering cross sections for the right- and lefthanded circularly polarized scattered light, respectively [see Fig. 1(a)]. Integrating $\eta$ over the two angles, we define the total degree of helical polarization $\eta_{\text {tot }}=$ $(4 \pi)^{-1} \iint \sin (\theta) \eta(\varphi, \theta) d \theta d \varphi \in[-1,1]$. We will use these 
quantities to determine and illustrate the conservation of helicity in scattering processes.

Having introduced the general properties of the incident beam, we now proceed to consider the so-called dual scatterers. A particular example of a dual scatterer can be achieved with nanoparticles with electric and magnetic dipolar polarizabilities $\left(\alpha_{e}\right.$ and $\left.\alpha_{m}\right)$ identical both in amplitude and in phase. In Fig. 1(b), we plot the electric and magnetic polarizabilities of such a scatterer-the $230-\mathrm{nm}$ radius silicon $(n=3.5)$ sphere. Polarizabilities are equal for wavelengths of $\lambda=1844 \mathrm{~nm}$ and $1160 \mathrm{~nm}$, marked with the vertical dashed blue lines. Note that for the latter case, the scattering spectrum is dominated by higher-order modes, and we cannot consider the spheres as dipolar scatterers. Furthermore, the spectra of $\left|\alpha_{e}\right|^{2}$ and $\left|\alpha_{m}\right|^{2}$ cross also at $\lambda=1520 \mathrm{~nm}$, but for this wavelength the phases of the polarizabilities are different (i.e., $\operatorname{Re}\left\{\alpha_{e}\right\}=-\operatorname{Re}\left\{\alpha_{m}\right\}$, which corresponds to the almost-zero-forward condition [23-25]). Polarizabilities and the scattering cross section plotted in Fig. 1(b) have been obtained from the Mie theory.

In the spectral range where the scattered fields can be described by dipolar electric and magnetic responses, the polarization-resolved differential cross section takes the following, analytical form [34]:

$$
I_{\mathbf{\epsilon}}(\mathbf{n}) \propto\left|\mathbf{\epsilon}^{*} \cdot \mathbf{p}+\left(\mathbf{n} \times \mathbf{\epsilon}^{*}\right) \cdot \mathbf{m} / c\right|^{2},
$$

with $\mathbf{\epsilon}$ corresponding to the polarization of the scattered light, and $\mathbf{p}$ and $\mathbf{m}$ denoting the electric and magnetic dipoles, respectively. The dipoles are induced by the incident fields $(\mathbf{E}, \mathbf{H})$,

$$
\mathbf{p}=\varepsilon_{0} \alpha_{e} \mathbf{E}, \quad \mathbf{m}=\alpha_{m} \mathbf{H} .
$$

For the dual nanoparticle $\left(\alpha_{e}=\alpha_{m}=\alpha_{0}\right)$, using the relationship Eq. (2), we get

$$
I_{\mathbf{\epsilon}}(\mathbf{n}) \propto\left|\varepsilon_{0} \alpha_{0}^{*} \mathbf{E}^{*} \cdot[\mathbf{\epsilon}+i \Lambda \mathbf{n} \times \mathbf{\epsilon}]\right|^{2} .
$$

We want to investigate the polarization of the scattered light in the basis of right-handed circularly polarized $\mathbf{r}(\mathrm{RC})$ and left-handed circularly polarized $\mathbf{l}$ (LC) light. For any scattering direction $\mathbf{n}$, we have $\mathbf{n} \times \mathbf{l}=-i \mathbf{l}$ and $\mathbf{n} \times \mathbf{r}=i \mathbf{r}$. Thus, the squared expression in the scattering cross section [Eq. (6)] is proportional to

$$
\left(\begin{array}{c}
I_{\mathrm{LC}} \\
I_{\mathrm{RC}}
\end{array}\right) \propto\left|\left(\begin{array}{l}
\mathbf{l} \\
\mathbf{r}
\end{array}\right)+\Lambda\left(\begin{array}{c}
\mathbf{1} \\
-\mathbf{r}
\end{array}\right)\right|^{2},
$$

indicating that for the $\Lambda=1(-1)$ incident beams, the $\mathrm{RC}$ (LC) polarization of the scattered light vanishes. Therefore, for the dual nanoparticle and the incident helical field, $\eta(\mathbf{n})$ for every direction should be equal to 1 , giving $\eta_{\text {tot }}=$ $1(-1)$ for the incident $\Lambda=-1(1)$ light.

We illustrate this scattering invariant in Fig. 1(c). In the plots, we present the spectra of the total degree of helical polarization $\eta_{\text {tot }}$, calculated for the scatterer positioned on the axis of the beam (top panel), or shifted away from it by $d=2 \mu \mathrm{m}$ (middle panel) or $d=4 \mu \mathrm{m}$ (bottom panel), as shown schematically in the insets. For the two wavelengths at which the two polarizabilities match $(\lambda=1160 \mathrm{~nm}$ and $1844 \mathrm{~nm}), \eta_{\text {tot }}$ reaches its maximum value 1 , indicating a fully circular polarization of the scattered light.

The scattering of a dual nanoparticle preserves the helicity everywhere and not only the far-field components as we have shown. As the scattered near field, composed primarily of evanescent waves, is essential for the understanding of systems comprised of many scatterers, below we will investigate in detail the helicity of the entirety of the scattered field for the dipolar spheres.

To arrive at this result, we consider the relationship between the electric $\mathbf{p}$ and magnetic $\mathbf{m}$ dipoles induced in a dual nanoparticle by helical light. Inserting Eq. (2) into definitions given in Eq. (5), we arrive at

$$
\mathbf{m}=-i c \Lambda \mathbf{p} .
$$

The scattered electric field from such a pair of dipoles can be expressed through Green's functions as

$$
\mathbf{E}_{\text {scatt }}=\frac{k^{2}}{\varepsilon_{0}} \mathbf{G}_{E} \mathbf{p}+i Z k^{2} \mathbf{G}_{M} \mathbf{m}=\frac{k^{2}}{\varepsilon_{0}}\left(\mathbf{G}_{E}+\Lambda \mathbf{G}_{M}\right) \mathbf{p} .
$$

To calculate the action of the helicity operator on $\mathbf{E}_{\text {scatt }}$, we use the following property of the Green's function;

$$
\Lambda \mathbf{G}_{\mathbf{E}}=\mathbf{G}_{\mathbf{M}}, \quad \mathbf{\Lambda} \mathbf{G}_{\mathbf{M}}=\mathbf{G}_{\mathbf{E}},
$$

which can be derived taking the definition of the helicity operator, Eq. (1). We then have

$$
\Lambda \mathbf{E}_{\text {scatt }}=\frac{k^{2}}{\varepsilon_{0}}\left(\mathbf{G}_{M}+\Lambda \mathbf{G}_{E}\right) \mathbf{p} .
$$

Since the eigenvalues of the helicity operator follow $\Lambda^{2}=1$, we can rewrite the above equations as

$$
\Lambda \mathbf{E}_{\text {scatt }}=\Lambda \frac{k^{2}}{\varepsilon_{0}}\left(\Lambda \mathbf{G}_{M}+\mathbf{G}_{E}\right) \mathbf{p}=\Lambda \mathbf{E}_{\text {scatt }} .
$$

This result is not so surprising if we consider that Eq. (8) represents the only two pairs of dipoles which produce a field with the well defined helicity $\Lambda$ [35]. Then, when the dipolar moments dominate, Eq. (8) ensures that the helicity is preserved everywhere (near and far field).

Since the helicity of the electromagnetic field is conserved in the process of scattering on a single dual scatterer, it should also be conserved in the subsequent scattering events on other dual scatterers. To illustrate this helicity invariance, we consider the scattering of the helical beam on a dimer of the modeled silicon spheres [33]. The central panels in Fig. 2 show the distribution of the two quantities defined as

$$
\Lambda_{ \pm}=\left|\mathbf{E}_{\text {scatt }} \pm i c \mu_{0} \mathbf{H}_{\text {scatt }}\right|^{2},
$$




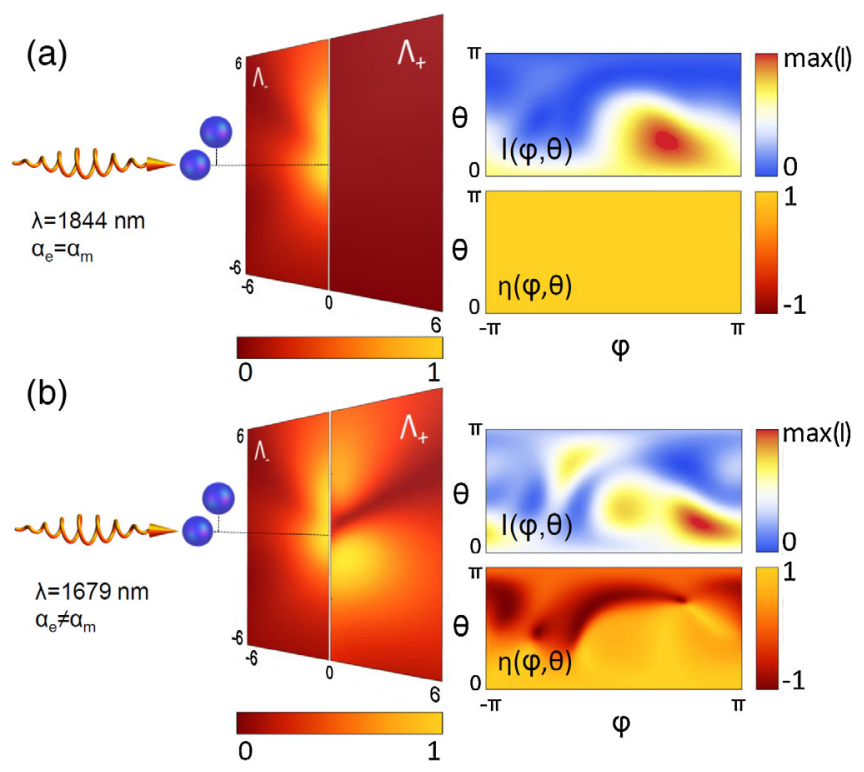

FIG. 2 (color online). Light scattering by a dimer of spheres. The incident helical $(\Lambda=-1)$ light of (a) $1844 \mathrm{~nm}$ or (b) $1679 \mathrm{~nm}$ wavelength is scattered on a dimer of two (a) dual and (b) nondual silicon spheres. One of the spheres is positioned at the origin of the coordinate system and the other is shifted from it $0.5 \mu \mathrm{m}$ along the axis and $1 \mu \mathrm{m}$ in the transverse direction. In the near field (calculated at $z=3 \mu \mathrm{m}$ ), the helicities of the scattered light can be measured by calculating distributions $\Lambda_{+}$ (central panels). Vanishing distribution of $\Lambda_{+}$in (a) indicates that the scattered light retains the negative helicity of the incident light, while the nonvanishing distributions in (b) indicate mixing of the $\Lambda=-1$ and $\Lambda=1$ components of light. Right panels represent the differential scattering cross sections of the scattered light $I(\varphi, \theta)$ (upper plots) and of the polarization degree $\eta(\varphi, \theta)$ (lower panel). For the dual spheres (a) $\eta(\varphi, \theta)=1$ is a constant function, indicating that the scattered light is fully circularly polarized $\left(\eta_{\mathrm{tot}}=1\right)$.

calculated in the transverse plane defined by $z=3 \mu \mathrm{m} . \Lambda_{ \pm}$ computes the scattered intensity into modes with helicity \pm . Two wavelengths of the incident light are considered: (a) $\lambda=1844 \mathrm{~nm}$, for which the scatterers are dual, and (b) $\lambda=1679 \mathrm{~nm}$, at which the scatterers have a dominating magnetic dipolar response. For a helical beam with $\Lambda=-1$ interacting with a dual dimer, the scattered intensity into modes with $\Lambda_{+}$will be zero as shown in Fig. 2(a). In contrast, for the nondual scatterers [Fig. 2(b)], neither one of the two fields $\Lambda_{ \pm}$vanishes, indicating the mixing of the two helicities in the scattering process. For both wavelengths, we also investigate the far-field properties of the scattered light, plotting its differential scattering cross section $I(\varphi, \theta)$ and the degree of helical polarization $\eta(\varphi, \theta)$ in the right panels of Fig. 2. The scattered light is shown to be fully circularly polarized only for the dual sphere [Fig. 2(a)].

We can also extend our considerations to the random media, modeled as a distribution of the dual scatterers, where each one preserves the helicity in a single scattering
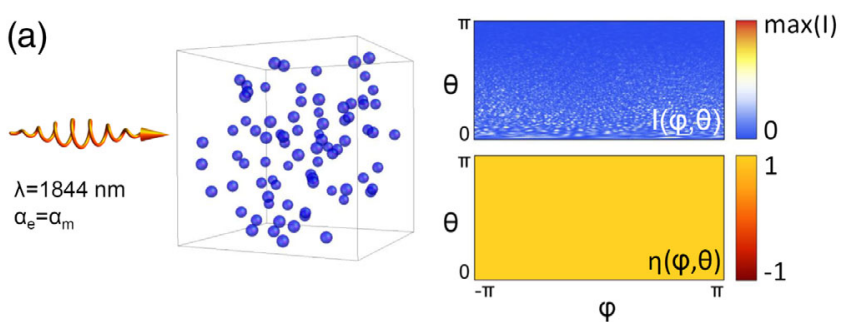

(b)
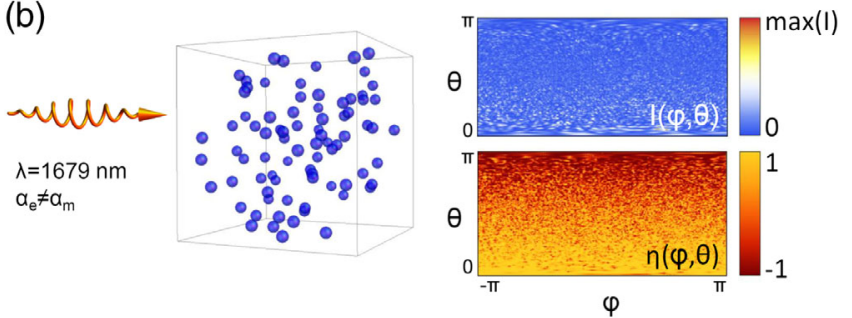

FIG. 3 (color online). Light scattering by a random medium. The incident helical $(\Lambda=-1)$ light of (a) $1844 \mathrm{~nm}$ or (b) $1679 \mathrm{~nm}$ wavelength scatters on an ensemble of 80 randomly distributed (a) dual and (b) nondual silicon spheres. The scatterers were randomly distributed in a cubic box with edges of $60 \mu \mathrm{m}$ length, centered on the axis of the beam. Panels on the right represent the differential scattering cross section $I(\varphi, \theta)$ (upper plots) and of the polarization degree $\eta(\varphi, \theta)$.

event. By using a coupled electric and magnetic dipole method [36-38], we illustrate this process in Fig. 3, where we investigate the scattering of incident helical light on a random distribution of 80 nanoparticles positioned randomly in a cubic volume of $60 \mu$ m edge length. Similarly, as in Fig. 2, we consider two wavelengths of incident light: (a) $1844 \mathrm{~nm}$, at which the scatterers are dual, and (b) $1679 \mathrm{~nm}$. In the former case, the circular polarization degree $\eta$ is constant and equal to 1 for any scattering direction [right bottom panel in (a)], indicating the conservation of helicity in the multiscattering process. For the nondual scatterers, polarization degree does not exhibit any significant preservation of helicity.

It is worth noticing that the intensity distribution for dual particles presents a clear asymmetry between forward and backward scattering. Due to the conservation of angular momentum and helicity, a complete suppression of backscattering (at $\theta=0$ ) is expected [29] for dual and axially symmetric samples. The partial (statistically averaged) axial symmetry particle distribution explains the observed results. Such an asymmetry is not observed for the nondual medium [Fig. 3(b)].

Finally, we briefly discuss the robustness of the helicity conservation against the deviation from the perfect duality condition of the scattering medium. Such deviation naturally arises when nanoparticles in the solution are not identical, but represent a distribution of radii with some standard deviation $\sigma$. We have carried out simulations of random media comprising scatterers with radii distributed around $r_{0}=0.23 \mu \mathrm{m}$. For $\sigma=0.02 r_{0}$, the integrated 
helicity degree is reduced to 0.85 while $\eta_{t o t} \approx 0.6$ for $\sigma=0.06 r_{0}$. A complete set of results and more elaborate discussion is included in the Supplemental Material [39].

In conclusion, we have investigated the problem of scattering of helical light by dielectric nanoparticles exhibiting strong electric and magnetic activity. At the Kerker condition, when both electric and magnetic polarizabilities are equal, the scattering preserves the helicity and polarization of light. We have shown this anomalous conservation of the scattering polarization in the case of a single nanoparticle, a dimer, and a random solution of dielectric nanoparticles. Our results open a pathway to exploit properties in random scattering media, including intriguing applications in random lasing [40,41], as well as to provide new possibilities to characterize magnetic optical properties of nanoscatterers [42].

M. K. S. and J. A. acknowledge funding from Project No. FIS2013-41184-P of the Spanish Ministry of Economy and Competitiveness, the Project No. ETORTEK IE14-393 NANOGUNE'14 of the Department of Industry of the Government of the Basque Country, Project No. IT756-13 of the Department of Education and Culture of the Basque Country, and Grant No. AP-2012-4204 from the Spanish Ministry of Education, Culture, and Sport. J. J. S. acknowledges financial support by the Spanish MINECO (Grant No. FIS2012-36113) and by IKERBASQUE. G. M.-T. is supported by the Australian Research Council (Grant No. FT110100924).

*mikolaj_schmidt@ehu.es †uanjo.saenz@uam.es

[1] J. Dainty, Laser speckle and related phenomena (SpringerVerlag, Berlin, 1984).

[2] J. W. Goodman, Speckle phenomena in optics: Theory and applications (Roberts \& Company, Greenwood Village, 2010).

[3] A. Carswell and S. Pal, Appl. Opt. 19, 4123 (1980).

[4] Y. Kuga and A. Ishimaru, J. Opt. Soc. Am. A 1, 831 (1984).

[5] M. P. Van Albada and A. Lagendijk, Phys. Rev. Lett. 55, 2692 (1985).

[6] P.-E. Wolf and G. Maret, Phys. Rev. Lett. 55, 2696 (1985).

[7] R. Lenke and G. Maret, Scattering in Polymeric and Colloidal Systems, edited by W. Brown and K. Mortensen, pp. 1-73 (Gordon and Breach, Amsterdam, 2000).

[8] G. Maret and P. Wolf, Z. Phys. B 65, 409 (1987).

[9] D. J. Pine, D. A. Weitz, P. M. Chaikin, and E. Herbolzheimer, Phys. Rev. Lett. 60, 1134 (1988).

[10] D. Weitz and D. Pine, Dynamic light scattering, edited by W. Brown (Oxford University Press, Oxford, 1993).

[11] M. Moscoso, J. B. Keller, and G. Papanicolaou, J. Opt. Soc. Am. A 18, 948 (2001).

[12] O. Angelsky, A. Ushenko, Y. A. Ushenko, and Y. G. Ushenko, J. Phys. D 39, 3547 (2006).

[13] A. Pierangelo, A. Benali, M.-R. Antonelli, T. Novikova, P. Validire, B. Gayet, and A. De Martino, Opt. Express 19, 1582 (2011).
[14] M. Zerrad, J. Sorrentini, G. Soriano, and C. Amra, Opt. Express 18, 15832 (2010).

[15] C. Schwartz and A. Dogariu, Opt. Lett. 31, 1121 (2006).

[16] F. Flossmann, K. O'Holleran, M. R. Dennis, and M. J. Padgett, Phys. Rev. Lett. 100, 203902 (2008).

[17] D. Bicout, C. Brosseau, A. S. Martinez, and J. M. Schmitt, Phys. Rev. E 49, 1767 (1994).

[18] E. Gorodnichev, A. Kuzovlev, and D. Rogozkin, JETP Lett. 68, 22 (1998).

[19] L. F. Rojas-Ochoa, D. Lacoste, R. Lenke, P. Schurtenberger, and F. Scheffold, J. Opt. Soc. Am. A 21, 1799 (2004).

[20] A. B. Evlyukhin, C. Reinhardt, A. Seidel, B. S. Lukyanchuk, and B. N. Chichkov, Phys. Rev. B 82, 045404 (2010).

[21] A. García-Etxarri, R. Gómez-Medina, L. S. Froufe-Pérez, C. López, L. Chantada, F. Scheffold, J. Aizpurua, M. NietoVesperinas, and J. Sáenz, Opt. Express 19, 4815 (2011).

[22] M. K. Schmidt, R. Esteban, J. J. Sáenz, I. Suárez-Lacalle, S. Mackowski, and J. Aizpurua, Opt. Express 20, 13636 (2012).

[23] M. Nieto-Vesperinas, R. Gomez-Medina, and J. J. Sáenz, J. Opt. Soc. Am. A 28, 54 (2011).

[24] R. Gómez-Medina, B. García-Cámara, I. Suárez-Lacalle, F. González, F. Moreno, M. Nieto-Vesperinas, and J. J. Sáenz, J. Nanophoton. 5, 053512 (2011).

[25] J.-M. Geffrin et al., Nat. Commun. 3, 1171 (2012).

[26] S. Person, M. Jain, Z. Lapin, J. J. Sáenz, G. Wicks, and L. Novotny, Nano Lett. 13, 1806 (2013).

[27] Y. H. Fu, A. I. Kuznetsov, A. E. Miroshnichenko, Y. F. Yu, and B. Lukyanchuk, Nat. Commun. 4, 1527 (2013).

[28] M. Kerker, D.-S. Wang, and C. Giles, J. Opt. Soc. Am. 73, 765 (1983).

[29] I. Fernandez-Corbaton, X. Zambrana-Puyalto, N. Tischler, X. Vidal, M. L. Juan, and G. Molina-Terriza, Phys. Rev. Lett. 111, 060401 (2013).

[30] I. Fernandez-Corbaton, X. Zambrana-Puyalto, and G. Molina-Terriza, Phys. Rev. A 86, 042103 (2012).

[31] X. Zambrana-Puyalto, X. Vidal, M. L. Juan, and G. MolinaTerriza, Opt. Express 21, 17520 (2013).

[32] X. Zambrana-Puyalto, I. Fernandez-Corbaton, M. Juan, X. Vidal, and G. Molina-Terriza, Opt. Lett. 38, 1857 (2013).

[33] P. Albella, M. A. Poyli, M. K. Schmidt, S. A. Maier, F. Moreno, J. J. Saenz, and J. Aizpurua, J. Phys. Chem. C 117, 13573 (2013).

[34] J. D. Jackson, Classical electrodynamics (Wiley, New York, 1962).

[35] I. Fernandez-Corbaton and G. Molina-Terriza, Phys. Rev. B 88, 085111 (2013).

[36] V. Markel, J. Mod. Opt. 40, 2281 (1993).

[37] G. W. Mulholland, C. F. Bohren, and K. A. Fuller, Langmuir 10, 2533 (1994).

[38] P. C. Chaumet and M. Nieto-Vesperinas, Phys. Rev. B 61, 14119 (2000).

[39] See Supplemental Material at http://link.aps.org/ supplemental/10.1103/PhysRevLett.114.113902 for simulation details for polydisperse size distributions.

[40] D. Wiersma, Nat. Phys. 4, 359 (2008).

[41] S. Gottardo, R. Sapienza, P. D. Garcia, A. Blanco, D. S. Wiersma, and C. López, Nat. Photonics 2, 429 (2008).

[42] S. Karaveli and R. Zia, Phys. Rev. Lett. 106, 193004 (2011). 